\title{
Patología orgánica
}

\author{
Parés, A.; Caballería, J. \\ Unidades de Alcohología y de Hepatología. ICMD. Institut d'Investigacions Biomèdiques August Pi i Sunyer (IDIBAPS). \\ Hospital Clínic. Barcelona.
}

Enviar correspondencia: Dr. Albert Parés. Unidades de Alcohología y Hepatología, Hospital Clínic. C/. Villarroel, 170. 08036 BARCELONA. Tel. 9322754 99. Fax 9345155 22. E-mail: pares@medicina.ub.es

\section{RESUMEN}

El consumo crónico de alcohol se acompaña de un amplio espectro de trastornos orgánicos, derivados de la acción directa de la oxidación del alcohol y de la producción de acetaldehído, o bien de las deficiencias nutricionales asociadas. La enfermedad hepática alcohólica es una de las alteraciones más frecuentes en los alcohólicos crónicos y se caracteriza por presentar desde lesiones leves como esteatosis hasta una cirrosis hepática. También son importantes las alteraciones neurológicas, de patogenia menos clara, pero relacionadas con la acción directa del alcohol y las deficiencias nutricionales. Asimismo, es frecuente que el alcohólico crónico tenga manifestaciones del tracto gastrointestinal y páncreas, asi como trastornos hematológicos, metabólicos y endocrinos. El alcoholismo crónico también se asocia a osteoporosis y osteopenia, al desarrollo de ciertos tipos de cáncer y a la fetopatía alcohólica.

Palabras clave: Patología orgánica, cirrosis alcohólica, consumo de alcohol, revisión, síndrome alcohólico fetal.

\section{SUMMARY}

Chronic alcohol intake results in a wide spectrum of organic disturbances derived from the alcohol oxidation and the subsequent generation of acetaldehyde, as well as from the nutritional deficiencies associated with alcohol intake. Alcoholic liver disease is the most prevalent organic abnormality observed in chronic alcoholic, ranging from fatty liver to established cirrhosis. Neurologic diseases are also frequent. Its pathogenesis is less clear, but it probably results from the direct toxic effect of alcohol as well as nutritional deficiencies. Moreover, alcoholic patients also may experience other diseases of the gastrointestinal tract and pancreas, as well as hematological, metabolic and endocrine disturbances. Chronic alcoholism is also associated with osteopenia and osteoporosis, the development of cancer and the fetal alcoholic syndrome.

Keywords: Organic pathology, alcohol abuse, alcoholic liver disease, alcoholic hepatitis, alcoholic cirrhosis, fetal alcohol syndrome.
$\mathbf{E}$ I consumo crónico de alcohol se asocia con la aparición enfermedades que afectan casi todos los órganos. Los mecanismos fisiopatológicos responsables de las lesiones orgánicas provienen en gran parte de la oxidación del alcohol y la producción de acetaldehído, de los efectos detergentes del propio alcohol sobre las membranas celulares y de la frecuente asociación con deficiencias nutricionales. Este artículo compendia la patología orgánica relacionada con el consumo excesivo de alcohol.

\section{ENFERMEDAD HEPÁTICA}

Desde la antigüedad se conoce la asociación entre el consumo de bebidas alcohólicas y el desarrollo de enfermedad hepática, aunque hasta hace unas décadas se consideraba 
que la enfermedad hepática era consecuencia de las deficiencias nutricionales que suelen asociarse al alcoholismo, más que debidas al efecto tóxico directo del alcohol. En la actualidad existen suficientes datos epidemiológicos, clínicos y experimentales que permiten afirmar que, aunque existan otros factores que pueden contribuir a sus efectos tóxicos, el consumo crónico de alcohol es el responsable del desarrollo de la lesión hepática. En este sentido existe una correlación lineal directa entre el consumo de alcohol per cápita y la mortalidad por cirrosis en una serie de paises occidentales (figura 1)

La mayor parte del alcohol absorbido es metabolizado en el hígado, donde sufre dos procesos oxidativos, mediante los cuales pasa a acetaldehído y posteriormente a acetato. El primer paso oxidativo se produce principalmente en el citoplasma del hepatocito y está catalizado por la enzima alcoholdeshidrogenasa. En una menor proporción el alcohol se oxida en los microsomas a través de una vía metabólica específica denominada sistema oxidativo microsomal para la oxidación del etanol. Las catalasas localizadas en los peroxisomas constituyen una tercera vía metabólica cuya importancia es escasa o nula. Las consecuencias de la oxidación del alcohol son la producción de acetaldehído y un desequilibrio redox, ya que se produce nicotinamida adenina dinuleótido reducidao (NADH) a partir de la nicotinamida adenina dinucleótido (NAD) que actúa como coenzima aceptando hidrogeniones. El segundo paso oxidativo consiste en la formación de acetato a partir del acetaldehído, acción catalizada por una acetaldehído deshidrogenasa con el concurso también de NAD que se reduce a NADH. La mayoría de efectos tóxicos del alcohol son debidos a la desproporción NADH/NAD y a la acción tóxica del acetaldehído (1).

Distintos estudios epidemiológicos se han verificado que sólo una proporción relativamente pequeña de alcohólicos tienen lesiones hepáticas intensas, a pesar de una duración y una magnitud de la ingesta alcohólica similar a la de los alcohólicos sin lesiones. Se deduce, por lo tanto, que existen otros factores, además del consumo crónico de alcohol, que influyen en el desarrollo de lesiones hepáticas. Entre los factores investigados destacan factores genéticos como el sexo, los antígenos de histocompatibilidad y la prevalencia de las distintas isoenzimas de la alcohol y de la aldehído deshidrogenasa (2), la malnutrición, alteraciones inmunológicas y los virus de la hepatitis (3).

\section{Figura 1. Correlación entre el consumo de alcohol y la mortalidad por cirrosis en los paises occidentales.}

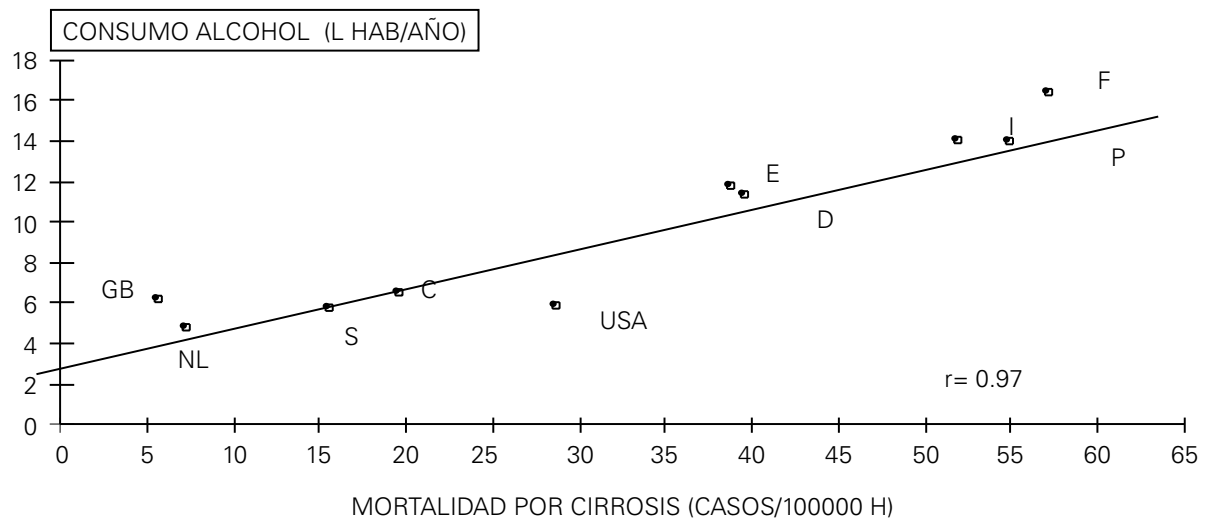


En el hígado el consumo crónico de alcohol se asocia con la aparición de diversas lesiones, que pueden observarse de forma individualizada, pero que en muchas ocasiones pueden coexistir. A continuación se describen las enfermedades hepáticas debidas a la ingesta excesiva de alcohol.

\section{Esteatosis}

El depósito de grasa en el citoplasma de los hepatocitos es la lesión más frecuente y se observa, en mayor o menor grado, en la mayoría de alcohólicos como lesión única o acompañando a otras alteraciones más graves. La esteatosis es la consecuencia de los cambios en el metabolismo de los lípidos ocasionados por el alcohol y se caracteriza por el depósito de grasa en vacuolas de distinto tamaño en el citoplasma de los hepatocitos. Estas vacuolas se van uniendo hasta formar una gran vacuola única que ocupa todo el citoplasma y desplaza al núcleo hacia la periferia de la célula. En ocasiones se asocia a daño celular y se produce una respuesta inflamatoria con participación de linfocitos y macrófagos, constituyendo los lipogranulomas. La esteatosis se localiza preferentemente en las áreas centrolobulillares.

La esteatosis microvesicular es una forma especial de esteatosis que se caracteriza por la presencia de múltiples vacuolas grasas de pequeño tamaño en el citoplasma que no suele desplazar al núcleo. Esta lesión se distribuye en los hepatocitos situados alrededor de la vena central y muchas veces se asocia a una hepatitis alcohólica o a una colestasis (4).

La esteatosis hepática aislada suele ser asintomática y manifestarse sólo por una hepatomegalia blanda y no dolorosa que disminuye de tamaño con la abstinencia. Las pruebas biológicas son inespecíficas. La alteración más constante es una elevación de la gammaglutamil transpeptidasa y un pequeño aumento de transaminasas. La ecografía abdominal muestra un hígado aumentado de tamaño con una estructura muy brillante. Algunos casos de esteatosis masiva pueden presentarse con manifestaciones de insufi- ciencia hepática grave, con descenso del tiempo de protrombina y encefalopatía hepática. Cuando la esteatosis se asocia a otras lesiones más avanzadas como fibrosis, hepatitis alcohólica o cirrosis, las manifestaciones clínicas que predominan son las propias de estas últimas lesiones. La esteatosis microvesicular alcohólica que, como se ha indicado, suele asociarse a lesiones de hepatitis alcohólica presenta una sintomatología inespecífica como astenia, anorexia, náuseas, vómitos y, en ocasiones, dolor abdominal. La hepatomegalia es constante y la ictericia frecuente. También es prácticamente constante el aumento de las concentraciones de colesterol y de triglicéridos en el suero. En la mitad de los casos existe una hiperbilirrubinemia conjugada y un descenso del tiempo de protrombina (4).

\section{Fibrosis}

La fibrosis hepática sin lesiones de hepatitis alcohólica, sin esteatosis y sin cirrosis no es una entidad clínico patológica suficientemente reconocida. Sin embargo, se ha observado que existe un número creciente de pacientes alcohólicos que presentan un aumento de colágeno hepático como única lesión histológica. Se considera que la fibrosis es secundaria a profundas alteraciones del metabolismo del colágeno que incluye un aumento de la síntesis o fibrogénesis y una disminución de la degradación o colagenolisis.

Las manifestaciones clínicas de la fibrosis hepática se caracterizan por trastornos generales inespecíficos y hepatomegalia. En la analítica destaca una hipertransaminasemia moderada. La evolución de la fibrosis hepática es poco conocida, pero se considera que la fibrosis pericelular y el engrosamiento de la pared de las venas terminales podrían tener un papel importante en la progresión a la cirrosis (5).

\section{Hepatitis alcohólica}

La hepatitis alcohólica se caracteriza por la existencia de áreas de necrosis celular, con 
un infiltrado inflamatorio constituido por leucocitos polimorfonucleares, en general de localización centrolobulillar. En estas áreas de necrosis los hepatocitos son grandes y presentan un citoplasma claro, en cuyo interior se observan agregados de una material homogéneo, intensamente acidófilo, de limites irregulares y de localización perinuclear, que reciben el nombre de hialina alcohólica o cuerpos de Mallory, y están constituidos por una agregación de fibrillas de naturaleza proteica. Estas lesiones pueden asociarse a esteatosis, fibrosis o cirrosis (6).

El espectro clínico de la hepatitis alcohólica es muy amplio y comprende desde formas asintomáticas hasta formas fulminantes con insuficiencia hepatocelular (7). El cuadro clínico que puede estar precedido por una temporada con una intensificación de la ingesta alcohólica, se inicia con astenia, anorexia, náuseas y vómitos. Al cabo de pocos días puede aparece dolor abdominal de predominio en hipocondrio derecho y epigastrio, ictericia y fiebre. La palpación del abdomen permite comprobar una hepatomegalia dolorosa. El resto de la exploración muestra, a menudo, otros estigmas de alcoholismo como hipertrofia parotídea, neuritis periférica, lengua carencial e, incluso, estigmas de hábito cirrótico. Los exámenes biológicos revelan signos discretos de insuficiencia hepatocelular, con transaminasas ligeramente elevadas, que rara vez sobrepasan las $300 \mathrm{U} / \mathrm{l}$. Casi siempre la aspartato aminotransferasa (AST) es superior a la alanino aminotransferasa (ALT) con un cociente AST/ALT habitualmente superior a 1.5. La gamma-glutamil transferasa (GGT) suele estar muy elevada. Asimismo por lo común están presentes signos de colestasis, con hiperbilirrubinemia conjugada y elevación de la fosfatasa alcalina. La anemia macrocítica es secundaria al alcoholismo o a carencias nutritivas. En la hepatitis alcohólica se observan con frecuencia trombocitopenia y leucocitosis con desviación a la izquierda. En estos casos hay que descartar una infección concomitante (7).

Las manifestaciones clínicas de la hepatitis alcohólica sugieren, a veces, un abdomen agudo como una colecistitis, una colangitis o un absceso hepático. La identificación del factor etiológico es importante para establecer el diagnóstico. En otros casos la hepatomegalia puede tener una superficie abollonada, donde se ausculta un soplo debido a la presencia de anastomosis arteriovenosas intrahepáticas y a un aumento del flujo arterial hepático. En estos casos debe efectuarse el diagnóstico diferencial con un carcinoma hepatocelular. En ocasiones la hepatitis alcohólica es totalmente asintomática y se diagnostica en pacientes alcohólicos que consultan por problemas extrahepáticos o que están ingresados para tratamiento de su dependencia alcohólica y a los que se practica una biopsia hepática debido a la presencia de alguna alteración biológica. Otras veces predominan las manifestaciones propias de una hepatopatía crónica, en particular las derivadas de una hipertensión portal, como ascitis o hemorragia digestiva por varices esofágicas. Con menor frecuencia la hepatitis alcohólica cursa con signos y síntomas de insuficiencia hepatocelular grave, falleciendo los pacientes a los pocos días con una insuficiencia renal progresiva. También de manera ocasional, la hepatitis alcohólica se asocia a esteatosis masiva, hemólisis e hiperlipemia transitoria, fundamentalmente a expensas de los triglicéridos, constituyendo el síndrome de Zieve.

\section{Hepatitis crónica}

Los alcohólicos pueden presentan lesiones semejantes a una hepatitis crónica de etiología vírica, pero el hecho de que se recuperen tras la supresión de la ingesta de alcohol es muy sugestivo de que el agente responsable de la enfermedad sea el alcohol. La hepatitis crónica que presentan los alcohólicos no tiene unas características clínicas definidas y, en general, se detecta al practicar una biopsia hepática. Las únicas diferencias analíticas de estos pacientes son unos niveles de ALT inferiores a los que se detectan en la hepatitis crónica por virus $\mathrm{C}$. Asimismo, es más frecuente que exista un aumento del volumen 
eritrocitario y del cociente AST/ALT y una menor concentración sérica de proteínas totales y de albúmina. Estas alteraciones se producirían como consecuencia del consumo crónico de alcohol. La detección sistemática de los anticuerpos antivirus $C$ permite delimitar mejor la responsabilidad del alcohol en estas hepatitis crónicas (8).

\section{Cirrosis}

La cirrosis alcohólica se caracteriza por la presencia de nódulos de regeneración rodeados de tejido fibroso que reemplazan a la estructura lobulillar normal. En los estadíos iniciales, los nódulos son uniformes, pequeños, de unos $3 \mathrm{~mm}$ de diámetro, aunque en las etapas finales el tamaño de los nódulos aumenta semejando una cirrosis macronodular como las de etiología vírica. La cirrosis puede asociarse a lesiones de hepatitis alcohólica.

Las manifestaciones clínicas de la cirrosis alcohólica son similares a las que presentan las cirrosis de otra etiología, junto a alteraciones ligadas al alcoholismo. En este sentido, son más evidentes los signos de desnutrición e hipovitaminosis, la hipertrofia parotídea y la retracción palmar de Dupuytren. También son frecuentes manifestaciones extrahepáticas propias del alcoholismo como polineuritis, trastornos de conducta o cuadros delirantes indicativos de un síndrome de abstinencia. Analíticamente existe una hipertransaminasemia y un aumento de la GGT, aunque los datos más sugestivos de cirrosis son el aumento de la gammaglobulina, el descenso del tiempo de protrombina y una trombopenia como reflejo del hiperesplenismo. La cirrosis puede permanecer asintomática, especialmente en los pacientes que dejan de beber. Cuando la enfermedad progresa aparecen los signos propios de hipertensión portal como ascitis, circulación colateral y varices esofágicas con la posibilidad de hemorragia digestiva. Cuando el grado de disfunción hepatocelular es muy marcado son frecuentes los episodios de encefalopatía hepática.

\section{Carcinoma hepatocelular}

Entre el $5 \%$ y el $15 \%$ de los pacientes con una cirrosis hepática alcohólica desarrollan un carcinoma hepatocelular. La causa de la degeneración neoplásica no es conocida y, aunque existen evidencias experimentales sobre el potencial carcinógeno del alcohol, es posible que los virus de la hepatitis tengan un papel fundamental en el desarrollo de muchos de estos hepatocarcinomas. En este sentido, se ha observado que existe una estrecha relación entre los anticuerpos frente al virus $\mathrm{C}$ y la presencia de un carcinoma hepatocelular en todo tipo de cirrosis. El desarrollo de un hepatocarcinoma debe sospecharse ante el deterioro rápido de un paciente con una cirrosis hepática. Actualmente el hepatocarcinoma puede detectarse en fases iniciales mediante la realización de ecografías periódicas.

\section{Pronóstico y tratamiento de la hepatopatía alcohólica}

La esteatosis hepática simple tiene un buen pronóstico, aunque la evolución puede ser menos favorable en aquellos pacientes que siguen bebiendo $y$, además, tienen otras lesiones como la fibrosis perivenular o la periportal. En algunos de estos casos se ha constatado, al repetir la biopsia, la progresión a la cirrosis. La mortalidad inmediata de la hepatitis alcohólica oscila entre el $10 \%$ y el $25 \%$ de los casos, dependiendo de la gravedad de los pacientes incluidos en cada serie. Los signos de mal pronóstico que comportan una elevada mortalidad durante el primer mes después del diagnóstico son una hiperbilirrubinemia superior a $12 \mathrm{mg} / \mathrm{dl}$, una tasa de protrombina inferior al 50\%, la encefalopatía hepática y la insuficiencia renal (7). La hepatitis alcohólica es una lesión precirrótica. En este sentido se ha demostrado que la persistencia de la ingesta de alcohol, la extensión de la lesión hepática y el sexo femenino son los factores de riesgo para la evolución hacia una cirrosis.

La cirrosis hepática compensada en los pacientes que dejan de beber tiene una supervivencia media acumulada relativamen- 
te prolongada que puede llegar a ser de casi diez años. Los signos de mal pronóstico son el aumento de la ictericia en ausencia de una hepatitis alcohólica, la presencia de ascitis refractaria al tratamiento diurético, la encefalopatía, la hemorragia digestiva por rotura de varices esofágicas y la insuficiencia renal funcional progresiva.

En el tratamiento de la hepatopatía alcohólica la primera medida a considerar es la abstinencia de alcohol. Con la abstinencia se consigue la curación de las lesiones iniciales como la esteatosis, mientras que en las formas más avanzadas es posible mejorar la supervivencia y retrasar la aparición de complicaciones. En este sentido la probabilidad de supervivencia en pacientes con hepatopatía alcohólica grave es significativamente superior en los pacientes que permanecen abstinentes que en aquellos que siguen bebiendo (figura 2). En las formas graves de hepatopatía alcohólica que requieren hospitalización es importante aplicar de manera precoz una serie de medidas generales como son la corrección de los trastornos hidroelectrolíticos, mejorar el estado nutricional, administrar preparados vitamínicos, tratar las infecciones y otras complicaciones y prevenir el síndrome de abstinencia (9).
En la hepatitis alcohólica se han ensayado diversos tratamientos específicos: glucocorticoides, esteroides anabolizantes, nutrición con aminoácidos, antitiroideos, hormonas hepatotróficas (insulina y glucagón), antifibrogénicos (colchicina, d-penicilamina), cianidanol y silimarina. Los resultados obtenidos hasta el momento con la mayoría de ellos son negativos o contradictorios. Los únicos que han mostrado algún efecto son los glucocorticoides y los suplementos nutricionales. En este sentido, en los pacientes con una hepatitis alcohólica grave que no presentan contraindicaciones se recomienda el tratamiento con prednisona a la dosis de $40 \mathrm{mg}$ administrados por vía oral durante cuatro semanas, seguido de una pauta descendente durante otras dos semanas (20 y 10 mg/día, respectivamente). Asimismo, es conveniente que estos pacientes consuman al menos 30 $\mathrm{kcal} / \mathrm{kg}$ y $1 \mathrm{~g} / \mathrm{kg}$ de proteínas al día. Para ello deben administrarse suplementos nutricionales y, en caso necesario, instaurar una pauta de nutrición enteral (11) o parenteral. La-Sadenosilmetionina también tiene un cierto efecto favorable en la cirrosis alcohólica (12).

El tratamiento de la cirrosis hepática consiste en el tratamiento de sus complicaciones. Estudios experimentales efectuados en

Figura 2. Probabilidad de supervivencia de los pacientes con una hepatopatía alcohólica grave en relación a la abstinencia.

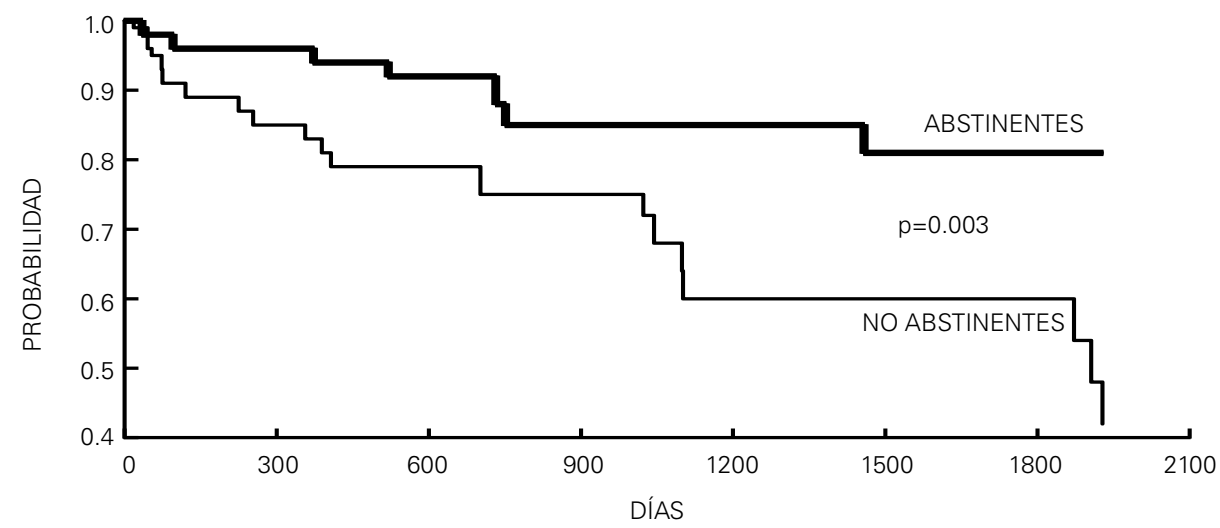


los últimos años han demostrado que algunas sustancias como la fosfatidilcolina son capaces de disminuir el daño hepático producido por el alcohol y retrasar el desarrollo de fibrosis.

El trasplante hepático es en la actualidad una opción terapéutica a tener en cuenta en la cirrosis alcohólica avanzada. La indicación de trasplante hepático en estos pacientes debe establecerse una vez transcurrido un periodo de abstinencia de al menos seis meses y en los pacientes que tengan conciencia clara de su dependencia del alcohol y un buen soporte sociofamiliar (13). Con una buena selección la supervivencia al año de estos pacientes es de alrededor del $80 \%$, similar a la de la cirrosis de otra etiología, con una tasa de recidiva de la ingesta del alcohol relativamente baja (14).

\section{PATOLOGÍA GASTROINTESTINAL}

El alcohol es absorbido en el estómago y en las primeras porciones del intestino, siendo muy elevada la concentración que se alcanza en el tracto gastrointestinal alto des- pués de la ingestión de alcohol, lo que favorece el desarrollo de lesiones en este tramo. Sin embargo, el alcohol puede producir lesiones en todo el aparato digestivo que se traducen en alteraciones tanto morfológicas como funcionales de la mucosa (15) (tabla 1).

\section{Esófago}

La administración de alcohol, tanto aguda como crónica, produce alteraciones motoras del esófago caracterizadas por una disminución de la presión de los esfínteres esofágicos superior e inferior y una disminución de la frecuencia de la peristalsis y de la amplitud de las ondas peristálticas, sin cambios en la velocidad de propagación. Estos cambios se observan preferentemente en alcohólicos crónicos que presentan una neuropatía periférica. Las consecuencias de esta disfunción motora son el reflujo gastroesofágico que condiciona la aparición de esofagitis y favorece el desarrollo de epitelio de Barrett, de estenosis pépticas y de úlceras del tercio inferior del esófago, todo lo cual aumenta la posibilidad de hemorragias. En los alcohólicos son frecuentes las náuseas y los vómi-

\section{Tabla 1. Efectos del alcohol sobre el tracto gastrointestinal.}

\section{Esófago}

- Disfunción motora con disminución de la presión de los esfínteres

- Esofagitis por reflujo y sus complicaciones

- Cáncer de esófago

\section{Estómago}

- Gastritis aguda

- Gastritis crónica

- Retraso en el vaciamiento gástrico

\section{Intestino delgado}

- Alteraciones de la mucosa duodenal y yeyunal

- Menor actividad de las enzimas de la mucosa

- Cambios en la actividad motora

- Alteración de los mecanismos de transporte

- Malabsorción 
tos, lo que puede ocasionar la aparición del síndrome de Mallory-Weiss, que es una causa de hemorragia digestiva.

Estudios epidemiológicos han hallado una asociación entre el consumo de alcohol y el cáncer de esófago, especialmente en los pacientes que además son fumadores. En estos casos el alcohol puede actuar como carcinógeno, vehiculizar al agente cancerígeno presente en el tabaco o alterar la mucosa esofágica haciéndola más vulnerable a otros carcinógenos. En la patogenia del cáncer de esófago también pueden intervenir factores nutricionales, como las deficiencias de hierro, cinc y vitamina $A$, que son muy frecuentes en los alcohólicos.

\section{Estómago}

La gastritis es consecuencia de la rotura de la barrera mucosa gástrica originada por el alcohol. La gastritis aguda después de una ingestión importante de alcohol es muy frecuente y revierte al cabo de unos días de abstinencia. El diagnóstico se efectúa por fibrogastroscopia y las lesiones endoscópicas características son mucosa eritematosa, erosiones, petequias y hemorragias. La gravedad de la gastritis se relaciona con la intensidad de las alteraciones de la barrera mucosa y con la concentración de ácido que entra en contacto con la mucosa. La ingestión simultánea de medicación potencialmente hemorragípara como ácido acetilsalicílico o antiinflamatorios no esteroideos, también favorece la intensidad de la gastritis alcohólica. La ingestión crónica de alcohol da lugar a gastritis crónica, tanto a la variedad superficial como a la gastritis crónica atrófica con hipoclorhidria, que es la forma más frecuente. También se ha referido una alta prevalencia de infección por Helicobacter pylori entre los alcohólicos crónicos lo que favorecería el desarrollo de gastritis crónica (16).

La rotura de la barrera mucosa gástrica da lugar a la retrodifusión de iones hidrógeno que ocasiona el daño celular. Los mecanismos que se han invocado como causantes de las alteraciones de la barrera mucosa gástrica son una disminución de la producción de moco, cambios en el flujo sanguíneo de la mucosa, una inhibición del transporte activo de iones, un aumento de la permeabilidad celular, la rotura de la membrana celular, hiperosmolaridad, una disminución de la concentración intracelular de prostaglandinas y una disminución del AMP cíclico (17). Posiblemente el daño de la mucosa gástrica se deba a una combinación de varios de estos mecanismos. A pesar de estos efectos deletéreos sobre la mucosa gástrica, no existen evidencias de una mayor incidencia de úlcera péptica entre los alcohólicos crónicos.

Los efectos del alcohol sobre la motilidad y el vaciamiento gástricos son contradictorios y parecen depender de la dosis. La administración de una dosis baja de alcohol acelera el vaciamiento gástrico, mientras que la administración de dosis altas o a mayor concentración disminuye la motilidad y retrasa el vaciamiento gástrico. Las alteraciones de la motilidad gástrica dependen de la existencia de una neuropatía periférica, de manera que en los alcohólicos crónicos sin neuropatía el vaciamiento gástrico es normal.

\section{Intestino delgado}

En animales de experimentación la administración intragástrica de alcohol a una concentración similar a la que tienen las bebidas alcohólicas produce erosiones y pequeñas hemorragias en las vellosidades intestinales que aparecen a los pocos minutos y remiten al cabo de unas horas. La intensidad de estas lesiones se correlaciona con la concentración del alcohol ingerido y con la proximidad del segmento estudiado. Lesiones similares se han observado en voluntarios sanos despues de ingerir una dosis única de alcohol (18). La ingestión crónica de alcohol se acompaña del acortamiento y la disminución del número de células diferenciadas de las vellosidades yeyunales y de un aumento de las células proliferativas de las criptas. Una menor actividad de las disacaridasas de la mucosa, un 
aumento de la permeabilidad del agua y otros solutos y cambios en la motilidad intestinal son los mecanismos causantes de la diarrea que, con frecuencia, presentan los alcohólicos crónicos. Las alteraciones estructurales del intestino delgado son las responsables de la malabsorción de distintos nutrientes como d-xylosa, la glucosa, aminoácidos, ácido fólico y otras vitaminas y minerales. Por el contrario, el alcohol no afecta directamente la absorción de grasas. La malnutrición y las alteraciones hepáticas y pancreáticas asociadas al alcoholismo crónico son las responsables de la esteatorrea (19).

\section{PATOLOGÍA PANCREÁTICA}

Diversos estudios epidemiológicos han demostrado la existencia de una estrecha relación entre el consumo excesivo de alcohol y la pancreatitis crónica (20). El alcohol generalmente da lugar a una pancreatitis crónica, recurrente y calcificante, pero se requiere un periodo de abuso de alcohol entre 6 y 12 años para que aparezcan los primeros síntomas de pancreatitis. El mecanismo patogénico de la pancreatitis alcohólica no es bien conocido. Clásicamente se ha invocado una disfunción del esfínter de Oddi en la patogenia de la pancreatitis alcohólica. En este sentido, existe una teoría que sugiere que el alcohol disminuiría el tono del esfínter de Oddi, facilitando el reflujo del contenido duodenal al páncreas. La enterocinasa presente en el jugo duodenal activaría a las enzimas pancreáticas en el interior de la glándula y daría lugar a la pancreatitis. Por el contrario, otra teoría propugna que el alcohol produciría un espasmo del esfínter, facilitando la comunicación entre la vía biliar y los conductos pancreáticos. Este hecho daría lugar al paso de bilis al páncreas, lo que causaría la pancreatitis. Estas teorías son muy discutibles ya que las evidencias experimentales que las sustentan son poco convincentes. El alcoholismo crónico también produce cambios en la secreción pancreática, produciendo un aumento en la concentración de proteínas que formarían precipitados en los conductos pancreáticos y darían lugar a la formación de trombos que, a su vez, ocasionarían la obstrución, inflamación y necrosis de la zona drenada por estos conductillos. Estos trombos podrían calcificarse y afectar a conductos pancreáticos de mayor calibre. Lo mas sugerente de esta teoría es que puede aplicarse a cualquier tipo de pancreatitis crónica, aunque no existen evidencias claras de que los precipitados de proteínas sean la causa y no la consecuencia de los cambios metabólicos que se producen durante la pancreatitis. El alcohol también produce cambios en la composición y fluidez de las membranas que pueden contribuir a la patogenia de la pancreatitis. La influencia de la dieta en la patogenia de la pancreatitis no está suficientemente aclarada. El papel de la malnutrición en la patogenia de la pancreatitis se apoya en el hecho de que los pacientes con una pancreatitis alcohólica presentan signos de malnutrición y en la observación de que los cambios en la secreción pancreática se normalizan con una dieta correcta, incluso con la persistencia de la ingesta de alcohol. Por otra parte, algunos estudios clínicos demuestran una elevada ingesta de proteínas y de grasa en los pacientes con pancreatitis alcohólica.

En los alcohólicos crónicos los síntomas de pancreatitis aguda generalmente aparecen cuando ya están bien establecidos los cambios funcionales e histológicos de pancreatitis crónica. El cuadro clínico se caracteriza por episodios recurrentes de dolor abdominal precipitados por un aumento de la ingesta habitual de alcohol. Cuando la enfermedad progresa los episodios dolorosos suelen ser mas frecuentes pero menos intensos, predominando las complicaciones como malabsorción, formación de seudoquistes, ictericia y diabetes. En algunos pacientes el curso clínico es muy insidioso y aparecen los signos de insuficiencia pancreática sin el antecedente de episodios agudos. El diagnóstico de pancreatitis crónica se efectúa por pruebas de función pancreática y por técnicas de imagen. Las técnicas de imagen utilizadas son la 
ultrasonografía, la tomografía axial computarizada y la colangiopancreatografía retrógrada endoscópica.

El carcinoma de páncreas es más frecuente en pacientes alcohólicos, aunque se correlaciona mejor con el consumo de tabaco que con el consumo de alcohol. La mayoría de pacientes con un carcinoma de páncreas también tienen lesiones de pancreatitis crónica, por lo que su diagnóstico puede ser difícil (21). Aparte de las técnicas de imagen habituales, la ultrasonografía endoscópica puede ayudar al diagnóstico de carcinoma de páncreas.

\section{PATOLOGÍA DEL SISTEMA NERVIOSO}

El consumo excesivo de alcohol, tanto agudo como crónico, así como su interrupción brusca produce diversos síndromes neurológicos, que son debidos a efectos tóxicos del etanol y del acetaldehído y a deficiencias nutricionales $(22,23)$ (tabla 2 ). El mecanismo de la toxicidad del alcohol no es bien conocido, aunque se cree que los cambios en la composición proteica y lipídica de las membranas desempeñan un papel importante.

\section{Intoxicación alcohólica}

Los síntomas de la intoxicación alcohólica reflejan una depresión de las funciones de las neuronas del sistema nervioso central. De acuerdo con el grado de intoxicación los síntomas mas comunes son excitación, desinhibición, verborrea, alteraciones de la conducta, incoordinación de movimientos y de la marcha, irritabilidad, estupor y coma. La cantidad de alcohol necesaria para producir intoxicación varía mucho de una persona a otra y depende, entre otros factores, del hábito alcohólico, del sexo y de la edad. Una intoxicación leve o moderada no requiere un tratamiento especial, mientras que el coma etílico constituye una urgencia médica grave, especialmente por la posible depresión de la función respiratoria.

\section{Síndrome de abstinencia alcohólica}

El síndrome de abstinencia alcohólica es la consecuencia de la dependencia física al alcohol. Las principales manifestaciones clínicas del síndrome de abstinencia son temblores, alucinaciones, convulsiones y delirio. El síntoma más frecuente son los temblores que aparecen a las pocas horas de la abstinencia y afectan sobre todo a manos, labios y lengua, y suelen acompañarse de nauseas, diaforesis, debilidad e irritabilidad. Entre las 24 y 48 horas del cese de la ingesta alcohólica aparecen alucinaciones visuales o auditi-

\section{Tabla 2. Enfermedades neurológicas producidas por el alcohol}

\section{Intoxicación alcohólica}

\section{Síndrome de abstinencia}

\section{Enfermedades relacionadas con trastornos nutricionales}

- Síndrome de Wernicke-Korsakoff

- Degeneración cerebelosa alcohólica

- Polineuropatía alcohólica

- Neuritis óptica

\section{Enfermedades de patogenia desconocida}

- Síndrome de Marchiafava-Bignani

- Mielinolisis pontina central

- Demencia alcohólica 
vas, que posteriormente suelen desaparecer en pocos días. Sin embargo, algunos pacientes pueden tener alucinaciones auditivas durante un tiempo prolongado y desarrollar un cuadro clínico similar a la esquizofrenia. El alcohol puede precipitar una crisis en pacientes epilépticos en el curso de una ingesta importante y ocasionar convulsiones en pacientes no epilépticos en la fase inicial del periodo de abstinencia. El delirium tremens es una manifestación grave del síndrome de abstinencia caracterizado por confusión, alucinaciones, temblores, agitación, taquicardia, pupilas dilatadas, sudoración profusa y fiebre. Los pacientes se recuperan al cabo de unos días, aunque en los pacientes que presentan una patología asociada como una enfermedad hepática o pancreática, traumatismos o neumonía por aspiración pueden tener mal pronóstico.

\section{Síndrome de Wernicke-Korsakoff}

El síndrome de Wernicke-Korsakoff es un trastorno nutricional causado por una deficiencia de tiamina (24). La encefalopatía de Wernicke representa la fase aguda, mientras que la psicosis de Korsakoff es la fase crónica. La encefalopatía de Wernicke se caracteriza por trastornos oculomotores, ataxia cerebelosa y confusión mental. Los trastornos oculomotores son nistagmus, diversos tipos de parálisis oculares u oftalmoplejia total. La ataxia afecta al tronco y a las extremidades inferiores. La confusión mental se caracteriza por desorientación, falta de atención y mala capacidad de respuesta. El estupor y el coma son frecuentes en las formas no diagnosticadas de encefalopatía de Wernicke y que no reciben tratamiento con tiamina por vía parenteral. La mayoría de pacientes que no se recuperan en 48-72 horas desarrollan psicosis de Korsakoff que se caracteriza por diversos grados de amnesia anterógrada y retrógrada, con conservación relativa de otras funciones intelectuales. La enfermedad es potencialmente reversible mediante tratamiento precoz con tiamina, pero en más del $50 \%$ de los casos la recuperación es incompleta.

\section{Degeneración cerebelosa alcohólica}

La degeneración de la corteza cerebelosa, especialmente del vermis anterior y superior y de las áreas hemisféricas adyacentes, es un hallazgo frecuente en las autopsias de alcohólicos crónicos. La enfermedad es más frecuente en hombres que en mujeres y se caracteriza por una ataxia del tronco y extremidades inferiores que condiciona una marcha inestable con conservación de la coordinación de las extremidades superiores. También puede observarse nistagmus, disartria y temblores. La enfermedad evoluciona lentamente a lo largo de años y puede mejorar con un aporte nutricional adecuado y el cese de la ingesta de alcohol. La práctica de una tomografía axial computarizada o de una resonancia nuclear magnética pude ayudar a confirmar la sospecha clínica. La patogenia de la degeneración cerebelosa es desconocida, aunque se ha atribuido al déficit nutricional y los efectos tóxicos directos del alcohol y del acetaldehído.

\section{Síndrome de Marchiafava-Bignani}

El síndrome de Marchiafava-Bignani consiste en una desmielinización del cuerpo calloso. Es muy poco frecuente y que se observa casi exclusivamente en alcohólicos crónicos. Se caracteriza por síntomas de afectación bilateral de los lóbulos frontales y disfunción hemisférica bilateral. Entre ellos destacan alteraciones del lenguaje, trastornos de la marcha, aumento del tono muscular e incontinencia urinaria. Los pacientes pueden presentar, además, trastornos de conciencia, temblores, convulsiones, afasia, hemiparesia y otros trastornos motores. El curso es progresivo hasta la muerte que suele aparecer en pocos años. Se desconoce la patogenia de este síndrome, aunque su asociación con la mielinolisis pontina central o con la enfermedad de Wernicke sugiere la existencia de un factor nutricional común.

\section{Mielinolisis pontina central}

La mielinolisis pontina central es una enfermedad desmielinizante rara que se caracteri- 
za por una disfunción neuronal localizada en la protuberancia. Las manifestaciones clínicas consisten en cuadriparesia progresiva, parálisis seudobulbar y parálisis parcial o completa de los movimientos horizontales del ojo. La enfermedad tiene a menudo una evolución fatal en 2 o 3 semanas y el diagnóstico con frecuencia no se efectúa antes del fallecimiento. La patogenia de este proceso es desconocida, aunque se ha sugerido que pueden desempeñar un papel los cambios bruscos en los niveles séricos de sodio producidos al corregir los desequilibrios electrolíticos y la pérdida de agua. No se dispone de un tratamiento específico.

\section{Polineuropatía alcohólica}

La polineuropatía es probablemente la complicación nutricional mas común que afecta el sistema nervioso de los alcohólicos (25). Los síntomas comienzan distalmente y siempre se afectan primero las extremidades inferiores, a menudo de forma exclusiva. El curso es insidioso y progresivo. Los pacientes aquejan dolor, parestesias y debilidad. En los casos graves se observan déficits motores distales y simétricos y atrofia. El reflejo aquíleo está disminuido o ausente, incluso en las fases asintomáticas. El diagnóstico clínico es claro y puede complementarse mediante electromiograma. El tratamiento consiste en la abstinencia alcohólica, administración de vitaminas del complejo B y mejoría del estado nutricional. A pesar de ello, la recuperación es lenta y a menudo incompleta.

\section{PATOLOGÍA MUSCULAR Y CARDÍACA}

\section{Miopatía alcohólica aguda}

La miopatía aguda alcohólica consiste en una necrosis muscular aguda que ocurre en bebedores muy importantes. La gravedad varía desde una rabdomiolisis con mioglobi- nuria a formas asintomáticas en las que sólo se observa una elevación transitoria de las enzimas musculares.

La rabdomiolisis aguda suele aparecer después de un episodio de ingesta importante y se caracteriza por dolor súbito, hinchazón y debilidad en uno o varios grupos musculares, elevación de la creatincinasa sérica, mioglobinuria y necrosis de las fibras musculares. El dolor dura unos pocos días, pero la debilidad puede persistir más tiempo. El diagnóstico se basa en la clínica, en la elevación de la creatincinasa, en la electromiografía y en la biopsia que muestra la necrosis de las fibras musculares. La complicación más grave es la insuficiencia renal aguda debida a una necrosis tubular provocada por la mioglobinuria. Sin embargo, en la mayoría de los casos el proceso es autolimitado y la recuperación se produce al cabo de unos días o semanas de abstinencia alcohólica.

\section{Miopatía alcohólica crónica}

Este tipo de miopatía se caracteriza por una progresiva debilidad y pérdida de masa muscular de los grupos musculares proximales, especialmente de las piernas. El cuadro suele ser indoloro, la mayoría de pacientes no tienen antecedentes de miopatía aguda y de manera casi constante se observa una neuropatía periférica asociada. El examen de la biopsia muscular muestra una atrofia selectiva de las fibras del tipo Ilb. La incidencia de este tipo de miopatía es desconocida ya que la atrofia de las fibras musculares tipo II es también frecuente en alcohólicos sin síntomas de enfermedad muscular (26).

Está bien demostrado el papel directo del alcohol o de sus metabolitos en la patogenia de la patología muscular. Sin embargo, no esta bien aclarado el papel concomitante de las deficiencias nutricionales, de los desequilibrios electrolíticos y de la isquemia. El tratamiento consiste en la abstinencia de alcohol, en corregir las alteraciones nutricionales y de los electrolitos y en tratar la patología renal asociada. 


\section{Miocardiopatía alcohólica}

La asociación entre el consumo excesivo de alcohol y la miocardiopatía congestiva se describió hace más de un siglo, aunque se atribuyó a déficits nutricionales o al efecto tóxico de ciertos aditivos. Sin embargo, en estudios posteriores en los que se excluyó a los alcohólicos malnutridos, ha podido demostrarse una relación directa entre el consumo de alcohol y las lesiones cardiacas (27).

La administración aguda de alcohol puede producir trastornos mecánicos y cambios electrofisiológicos en el corazón, efectos que suelen ser subclínicos. En los alcohólicos crónicos y en pacientes con una cardiopatía, la ingestión aguda de etanol puede producir alteraciones con traducción clínica.

El consumo crónico de alcohol puede conducir a una disfunción cardiaca progresiva que acaba en una miocardiopatía congestiva. Para ello se requiere un consumo excesivo de alcohol durante 10 o más años. El inicio del proceso es insidioso, con astenia, molestias torácicas, palpitaciones y algún episodio aislado de fibrilación auricular. Cuando la enfermedad progresa aparecen manifestaciones de insuficiencia cardiaca, derecha e izquierda. Así, puede haber ortopnea, disnea paroxística nocturna, ingurgitación yugular y edemas. Si persiste la ingesta alcohólica se produce la muerte por insuficiencia cardiaca o por sus complicaciones a los pocos años de haberse iniciado la clínica. También puede producirse la muerte súbita por fibrilación ventricular. En el electrocardiograma se observan diversas anomalías del espacio ST y de la onda $T$, ensanchamiento de las ondas $P$, bloqueo auriculoventricular, hemibloqueos y bloqueos de rama, y arritmias. En la ecocardiografía se puede demostrar una dilatación de las cuatro cavidades, hipertrofia ventricular izquierda y disminución de la función contráctil de ambos ventrículos. El cateterismo cardíaco revela una disminución del gasto cardíaco, elevación de las presiones de llenado de ambos ventrículos y descenso importante de los índices de contractilidad.
El tratamiento de la miocardiopatía alcohólica consiste en la abstinencia, combinada con el tratamiento de la insuficiencia cardíaca y de las arritmias acompañantes. El pronóstico está en relación con la duración y gravedad de los síntomas antes de iniciar la abstinencia.

\section{HIPERTENSIÓN ARTERIAL}

En la mayoría de estudios sobre la posible asociación entre el consumo de alcohol y la tensión arterial, se ha observado que en las personas con un consumo medio de tres a cuatro bebidas diarias, la tensión sistólica era 3 a $4 \mathrm{mmHg}$ superior a la de los no bebedores y la tensión diastólica, 1 a $2 \mathrm{mmHg}$ superior (28). Estas elevaciones son más marcadas en las personas que consumen de cinco a seis bebidas diarias, lo que demuestra una relación dosis-respuesta entre la tensión arterial y el consumo de alcohol. Se desconocen los mecanismos que pueden provocar esta elevación de la tensión arterial, aunque hay que considerar la efectos biológicos sobre la regulación cardiovascular, un efecto directo sobre el sistema nervioso central, la abstinencia y modificaciones en el metabolismo del calcio (29). Los alcohólicos que desarrollan un síndrome de abstinencia pueden presentar transitoriamente hipertensión importante, probablemente relacionada con la activación del sistema nervioso simpático y del sistema renina-angiotensina-aldosterona.

\section{PATOLOGÍA ENDOCRINA}

Los efectos gonadales del consumo de alcohol son frecuentes en los alcohólicos (30). Aunque estos efectos gonadales se han considerado como una consecuencia de la afectación hepática, está bien demostrado que el alcohol puede producir per se disfunción sexual, especialmente en los hombres, que experimentan hipoandrogenización con disminución de la líbido, impotencia o ambas 
cosas. La patogenia de estas anomalías no es bien conocida, aunque se ha observado que el alcohol o sus metabolitos pueden afectar directamente la función testicular al disminuir la producción de testosterona e interferir en la unión de la gonadotropina con el tejido testicular. Además, el alcohol puede influir sobre el eje hiopotálamo-hipófiso-gonadal. Los alcohólicos pueden presentar también hiperestrogenización que se manifiesta por eritema palmar, arañas vasculares y ginecomastia, junto con cambios bioquímicos como una aumento de la globulina fijadora de los esteroides sexuales. El alcohol puede inducir cambios en el metabolismo de la testosterona y de los estrógenos, así como alteraciones en los receptores de estos últimos, efecto que puede ser especialmente importante en los pacientes con una hepatopatía avanzada o con una anastómosis portosistémica. El alcohol también puede ser causa de infertilidad. En este sentido, se ha visto que el semen de los alcohólicos a menudo presenta anomalías, como un menor número y motilidad de los espermatozoides y una mayor proporción de formas anormales de éstos. Aunque es un hecho menos conocido, se sabe que el alcohol puede lesionar la arquitectura ovárica en ratas alimentadas con etanol, lo que puede explicar en parte la infertilidad de las mujeres alcohólicas.

Otra alteración endocrinológica observada en los alcohólicos crónicos es el "seudosíndrome de Cushing" (31) caracterizado por facies de luna llena, emaciación muscular, estrías abdominales, debilidad, astenia e hipertensión. Este síndrome puede ser indistinguible del verdadero síndrome de Cushing por la elevación de los niveles plasmáticos de cortisol y la incapacidad de la dexametasona de suprimir su secreción. Sin embargo, el "seudosíndrome de Cushing" desaparece con la abstinencia y puede reaparecer al reiniciar la ingestión de alcohol. Su patogenia parece relacionada con un efecto estimulador del alcohol sobre la secreción de cortisol, posiblemente mediado a través de la ACTH.

En los alcohólicos, especialmente en los que presentan una hepatopatía, también se han observado alteraciones de la hormona de crecimiento aunque carecen de relevancia clínica. También se ha descrito la presencia de niveles basales elevados de prolactina y una mayor respuesta de esta última a la hormona liberadora de tirotropina, efectos que son más destacados cuando existe una cirrosis hepática, circunstancia en la que también se ha observado una menor conversión de T4 a T3 y alteraciones de las proteínas fijadoras de tiroxina, aunque sin importancia clínica. La función paratiroidea, en general, no está afectada en los alcohólicos crónicos.

\section{TRASTORNOS METABÓLICOS}

\section{Hipoglucemia y cetoacidosis}

La hipoglucemia es una complicación poco común del abuso alcohólico. Suele aparecer después de un ayuno prolongado o en individuos gravemente mal nutridos tras la ingestión de una gran cantidad de etanol. Generalmente hay estupor y coma, y en algunas ocasiones se observan además otros hallazgos neurológicos, tales como desviación conjugada de la mirada, rigidez de las extremidades, convulsiones y reflejo de Babinski. La patogenia de esta hipoglucemia se atribuye principalmente a la inhibición de la gluconeogénesis hepática por el alcohol, en casos de notable agotamiento de los depósitos de glucógeno hepático. Además puede existir un trastorno de la secreción endógena de glucocorticoides.

La cetoacidosis alcohólica ocurre en los individuos que abusan crónicamente del alcohol, quienes después de un exceso importante y reciente desarrollan dolor abdominal y vómitos, que conducen a una situación de emaciación aguda. De hecho, en la mayoría de los pacientes con cetoacidosis alcohólica no se detectan niveles de alcoholemia debido a que han permanecido en ayuno durante más de 1 día. Los pacientes presentan taquipnea, aliento cetósico y alteraciones digestivas que, en general, reflejan la existen- 
cia de una gastritis aguda. Puede haber deshidratación y delirium tremens. El equilibrio ácido-base revela habitualmente una acidosis metabólica moderada, aunque el pH sanguíneo puede estar elevado a causa de la alcalosis respiratoria concomitante o de la alcalosis metabólica debida a los vómitos, o de ambas cosas. Los niveles séricos de cetonas están notablemente elevados, y puede haber un ligero aumento de las cifras séricas de lactato. La cetosis suele desaparecer al cabo de unas pocas horas de administrar glucosa y suero salino por vía intravenosa. La patogenia de este síndrome se atribuye a la interrupción de la ingesta alcohólica, lo que da lugar a la liberación de un bloqueo previo de la cetogénesis, inducido por el alcohol.

\section{Hiperuricemia}

El consumo de alcohol se ha relacionado con hiperuricemia y ataques de gota en los pacientes propensos a ello. El mecanismo de la hiperuricemia es una disminución en la excreción urinaria de ácido úrico, secundaria a hiperlactacidemia. El lactato inhibe competitivamente la eliminación del ácido úrico por el túbulo proximal renal y, por consiguiente, reduce la excreción de urato. Además, en los pacientes gotosos con mayor producción de ácido úrico, la ingesta alcohólica puede exacerbar la síntesis de urato por degradación acelerada de los nucleótidos de adenina.

\section{ALTERACIONES HEMATOLÓGICAS}

Las manifestaciones hematológicas son muy frecuentes en los alcohólicos crónicos con o sin afectación hepática. Estas anomalías se manifiestan sobre todo a los hematíes, pero también a los leucocitos y a las plaquetas (tabla 3). Asimismo, el consumo de alcohol produce algunas alteraciones en la hemostasia.

\section{Eritrocitos}

La mayoría de alcohólicos tienen un aumento del volumen corpuscular medio de los hematíes (32). Esta anomalía se asocia

\section{Tabla 3. Complicaciones hematológicas producidas por el alcohol}

\section{Hematíes}

- Efecto directo sobre la eritropoyesis

- Déficit de folato

- Alteraciones del metabolismo del hierro

\section{Hemólisis}

- Estructura anormal de las membranas

- Hipofosfatemia

\section{Leucocitos}

- Neutropenia

- Trastorno de la adherencia y de la quimiotaxis

- Trastorno de la función de los macrófagos

- Trastorno de la función de los linfocitos

\section{Plaquetas}

- Trombopenia

- Función anormal 
con el consumo de alcohol y suele desaparecer al cabo de unas semanas de abstinencia. Aunque el déficit de folato es frecuente en los alcohólicos, la macrocitosis simple no tiene relación con áquel y se considera que es una consecuencia del efecto tóxico directo del alcohol o de sus metabolitos sobre los eritroblastos en desarrollo que son vacuolados. Sin embargo, los alcohólicos pueden presentar déficits vitamínicos que dan lugar a megaloblastosis. Así, en los casos con un déficit nutricional de folato se observa megaloblastosis, aunque la vitamina B12 y la afectación gástrica e intestinal pueden desempeñar un papel en este tipo de anemia. En los alcohólicos con un déficit de folato también es frecuente una anemia sideroblástica, aunque la función anormal de la piridoxina o la disminución de la actividad de las enzimas que intervienen en la síntesis del hem, así como los trastornos del metabolismo del hierro pueden contribuir a este tipo de anemia.

Los alcohólicos pueden presentar anemias hemolíticas, aunque éstas son más frecuentes en los casos en que existe una hepatopatía grave. Se han descrito diversos síndromes hemolíticos en pacientes con una hepatopatía alcohólica debido a alteraciones de los hematíes o a la esplenomegalia secundaria a la enfermedad hepática y a la hipertensión portal. Cambios en la composición de los lípidos de la membrana eritrocitaria y en las lipoproteínas plasmáticas secundarios al consumo de alcohol, pueden disminuir la fluidez de estas membranas y dar lugar a acantocitosis. Se han descrito, además, anemias relacionadas con la presencia de dianocitos, estomatocitos y triangulocitos. Se desconoce la patogenia de estas anomalías morfológicas. Finalmente se ha observado anemia hemolítica en alcohólicos con hipofosfatemia marcada que puede ocasionar niveles bajos de adenosin trifosfato, necesario para mantener la actividad metabólica de los eritrocitos.

\section{Leucocitos}

En los alcohólicos puede haber granulocitopenia y trastornos de la adherencia, moviliza- ción y quimiotaxis de los granulocitos, así como alteraciones funcionales de los macrófagos y linfocitos que pueden explicar, en parte, la menor resistencia de estos sujetos a las infecciones. No se conoce bien la prevalencia de estas anomalías ni tampoco el papel respectivo que pueden desempeñar el alcohol, la malnutrición, la hepatopatía u otras enfermedades asociadas (33).

\section{Plaquetas}

La trombopenia es una consecuencia bien conocida de la ingesta alcohólica, aunque en la mayoría de los casos es poco importante y sin repercusiones clínicas. Generalmente la cifra de plaquetas se normaliza en el plazo de una semana después de haber cesado la ingesta de alcohol. El efecto sobre las plaquetas parece ser debido a una inhibición directa del alcohol sobre la trombocitopoyesis, ya que puede observarse trombopenia en ausencia de déficit de folato y de esplenomegalia (34). El etanol también puede ocasionar anomalías funcionales de las plaquetas, incluso en ausencia de trombopenia. En efecto, en los alcohólicos crónicos puede observarse un alargamiento del tiempo de hemorragia, un descenso de la agregación plaquetaria y una menor liberación de tromboxano A2 a partir de las plaquetas, anomalías que desaparecen al cabo de 1 a 3 semanas de abstinencia. No se conocen bien los mecanismos de la disfunción plaquetaria, aunque pueden estar relacionados con alteraciones en las funciones de las membranas de las plaquetas circulantes y de los megacariocitos.

\section{Hemostasia}

En los alcohólicos los factores de la coagulación suelen encontrarse dentro de los limites normales a menos de que exista una hepatopatía importante (35). Sin embargo, la antitrombina III puede estar disminuida en los alcohólicos crónicos, lo que puede explicar que se produzcan trombosis espontáneas en estos pacientes. Cuando los alcohólicos pre- 
sentan tiempos de hemorragia anormales se debe principalmente a trastornos de la función plaquetaria.

\section{OSTEOPENIA}

El consumo crónico de alcohol se ha relacionado con la aparición de osteopenia y osteoporosis, probablemente por un efecto directo del alcohol o del acetaldehído sobre la función osteoblástica. Durante muchos años se ha descrito una pérdida de masa ósea en los individuos alcohólicos, manifestada por una mayor incidencia de fracturas, aunque se consideró que los traumatismos y la asociación con una hepatopatía eran los factores primordiales. Sin embargo, estudios recientes indican que los alcohólicos pueden presentar osteopenia sin que haya una hepatopatía significativa, lo cual indica que el alcohol tiene un efecto tóxico directo sobre los osteoblastos y el remodelado óseo. Así pues, aunque los alcohólicos pueden presentar déficit nutricionales y malabsorción de calcio y de vitamina $D$ y anomalías de la función tiroidea, los bajos niveles de osteocalcina, que es un marcador sensible del recambio metabólico óseo, hallados en los alcohólicos sin hepatopatía indican que el alcohol puede ejercer una acción tóxica directa sobre los osteoblastos (36). El trastorno puede recuperarse parcialmente tras la abstinencia prolongada (37)

\section{ALCOHOLY CÁNCER}

La relación entre el consumo de alcohol y el cáncer se basa en estudios epidemiológicos que han demostrado una clara asociación entre el consumo excesivo de alcohol y el cáncer bucal, laríngeo y esofágico (38). Se ha observado que los alcohólicos tienen un riesgo 10 veces mayor de presentar cáncer bucal y laríngeo. En este tipo de cáncer es importante el efecto combinado del abuso de alco- hol y de tabaco, mientras que otros factores como las bebidas muy calientes, las deficiencias de vitaminas $A$ y $C$ y la mala higiene dental son menos importantes. El papel del alcohol en el desarrollo del cáncer esofágico es todavía más importante ya que el riesgo relativo aumenta logarítmicamente con el consumo creciente de alcohol, incluso cuando se corrigen los resultados de acuerdo con el consumo de tabaco. Los déficits de hierro, cinc y vitamina A también pueden estar implicados en la patogenia del cáncer esofágico. El cáncer hepático también se ha relacionado con el consumo de alcohol. La relación entre el alcohol y el cáncer de páncreas y de colon es menos clara, y en relación a esta última localización existen estudios contradictorios sobre la influencia del consumo de cerveza en el desarrollo de cáncer de recto. Existen varios estudios epidemiológicos que muestran una asociación entre el consumo de alcohol y el cáncer de mama (39), aunque otros autores consideran que aunque el riesgo de desarrollar un cáncer de mama entre los alcohólicos es mayor que el de la población no alcohólica, las diferencias son relativamente pequeñas. Dado que no existen pruebas de que el alcohol sea carcinógeno per se, puede haber otros mecanismos que contribuyan a la carcinogénesis. Así es posible que el alcohol aumente la susceptibilidad de diversos tejidos a los carcinógenos químicos, para lo cual puede activar dichos carcinógenos, alterar su metabolismo, inducir déficits nutricionales y modificar la respuesta inmunitaria. Además, hay que considerar la presencia de sustancias contaminantes carcinógenas en las bebidas alcohólicas.

\section{SÍNDROME ALCOHÓLICO FETAL}

El consumo crónico de alcohol durante el embarazo produce una serie de alteraciones en el desarrollo fetal que se manifiestan por un retraso en el crecimiento intrauterino que da lugar a recién nacidos de bajo peso, anomalías congénitas especialmente en boca y 
tracto genitourinario, irritabilidad, retraso intelectual y, en los casos más graves, el síndrome alcohólico fetal (40). Las anomalías craneofaciales incluyen alteraciones oculares como cavidades oculares pequeñas, ptosis palpebral y estrabismo, nariz pequeña con puente nasal ancho, y labio superior grande. Las alteraciones del sistema nervioso central incluyen numerosos defectos estructurales y funcionales cuyas consecuencias son microcefalia, hipoplasia del nervio óptico, disminución de la agudeza auditiva, dificultad de aprendizaje y retraso mental.

\section{BIBLIOGRAFIA}

(1) Lindros KO. Alcoholic liver disease: Pathobiological aspects. J Hepatol 1995; 23 (Suppl 1): 715.

(2) Parés X, Farrés J, Parés A, Soler X, Panés J, Ferré JL et al. Genetic polymorphism of liver alcohol dehydrogenase in Spanish subjects: Significance of alcohol consumption and liver disease. Alcohol Alcohol 1994; 29: 701-705.

(3) Parés A, Barrera JM, Caballería J, Ercilla G, Bruguera M, Caballería Ll et al. Hepatitis C virus antibodies in chronic alcoholic patients: Association with severity of liver injury. Hepatology 1990; 12: 1295-1299.

(4) Montull S, Parés A, Bruguera M, Caballería J, Uchida T, Rodés J. Alcoholic foamy degeneration in Spain. Prevalence and clinicopathological features. Liver 1989; 9: 79-85.

(5) Takada A, Nei J, Matsuda Y. Clinicopathological study of alcoholic fibrosis. Am J Gastroenterol 1982; 77: 660-666.

(6) MacSween RNM, Burt AD. Histological spectrum of alcoholic liver disease. Sem Liver Dis 1986; 6: 221-232.

(7) Parés A, Bosch J, Bruguera M, Rodés J. Características clínicas y criterios pronósticos de la hepatitis alcohólica. Gastroenterol Hepatol 1978: 1: 18-123.

(8) Parés A. Manifestaciones clínicas de la hepatopatía alcohólica. A. Parés. En "Actualidades en Gastroenterología y Hepatología". J. Rodés y C. Chantar Eds. Prous Editores, 1995.
(9) Mezey E. Treatment of alcoholic liver disease. Sem Liver Dis 1993; 13: 210-216.

(10) Cabré E, Rodríguez-Iglesias P, Caballería J, Quer JC, Sánchez-Lombraña JL, Parés A, et al. Short- and long-term outcome of severe alcohol-induced hepatitis treated with steroids or enteral nutrition: a multicenter randomized trial. Hepatology 2000;32:36-42.

(11) Mezey E, Caballeria J, Mitchell MC, Parés A, Herlong HF, Rodes J. Effect of parenteral amino acid supplementation on short-term and longterm outcomes in severe alcoholic hepatitis: a randomized controlled trial. Hepatology 1991; 14:1090-1096.

(12) Mato JM, Cámara J, Fernández J, et al. S-adenosylmethionine in the treatment of alcoholic liver cirrhosis: Results from a multicentric, placebo-controlled, randomized, double-blind clinical trial. Journal of Hepatology 1999;30:10811089.

(13) Hoofnagle JH, Kresina T, Fuller RK, Lake JR, Lucey MR, Sorrell MF et al. Liver transplantation for alcoholic liver disease: Executive statement and recommendations. Summary of a National Institutes of Health workshop held December 6-7, 1996, Bethesda, Maryland. Liver Transpl Surg 1997; 3: 347-350.

(14) Caballería J, Cugat E. El trasplante hepático en las hepatopatías alcohólicas. Medicina Interna 1991; 9: 560-567.

(15) Burbige EJ, Lewis DR Jr, Halsted CH. Alcohol and the gastrointestinal tract. Med Clin N Am 1984; 68: 77-89.

(16) Hauge T, Persson J, Kjerstadius T. Helicobacter pylori, actice chronic antral gastritis, and gastrointestinal symptoms in alcoholics. Alcoholism Clin Exp Res 1994; 18: 886-888.

(17) Feinman L, Korsten MA, Lieber CS. Alcohol and the digestive tract. En Lieber, CS (Ed). Medical and Nutritional Complications of Alcoholism. Mechanisms and Management. Plenum Medical Book Co., New York, 1992; 307340.

(18) Gottfried EB, Korsten MA, Lieber CS. Alcoholinduced gastric and duodenal lesions in man. Am J Gastroenterol 1978; 70: 587-592.

(19) Mezey E. Intestinal function in chronic alcoholism. Ann New York Acad Sci 1975; 252: 215227. 
(20) Singh M, Simsek H. Ethanol and the pancreas. Current status. Gastroenterology 1990; 98: 1051-1062.

(21) Gold EB, Goldin SB. Epidemiology for and risk factors for pancreatic cancer. Surg Oncol Clin N Am 1998; 7: 67-91.

(22) Charness ME, Simon RP, Greenberg DA. Ethanol and the nervous system. N Engl J Med 1989; 321: 442-454.

(23) Nicolás JM, Estruch R, Salamero M, Orteu N, Fernández-Solá J, Sacanella E,Urbano-Márquez A. Brain impairment in well-nourished chronic alcoholics is related to alcohol intake. Ann Neurol 1997; 41: 590-598.

(24) Butters N. Alcoholic Korsakoff's syndrome: an update. Sem Neurol 1984; 4: 226-244.

(25) Victor M. Neurologic disorders due to alcoholism and malnutrition. En Clinical Neurology, AB Baker y RJ Joynt eds, Philadelphia, Harper and Row, vol 4, 1986.

(26) Fernández-Solá, Junyent JM, Urbano-Márquez A. Alcoholic myopathies. Curr Opin Neurol 1996; 9: 400-405.

(27) Urbano-Márquez A, Estruch R, Navarro-López F, Grau JM, Mont L, Rubin E. The effects of alcoholism on skeletal and cardiac muscle. N Engl J Med 1989; 320: 409-415.

(28) Beilin LJ, Puddey IB. Alcohol and hypertension. Clin Exp Hyperten 1992;14:119-138.

(29) Coca A, Garay RP, Aguilera MT, De la Sierra A, Urbano-Márquez A. Disturbances of transmembranous sodium transport systems induced by ethanol in human erythrocytes. An approach to the pressor effect of alcohol. Am J Hypertens 1989; 2: 784-787.

(30) Gavaler JS, Van thiel DH. Reproductive consequences of alcohol abuse: Males and females compared and contrasted. Mut Res 1987; 186: 269-277.

(31) Jeffcoate W. Alcohol-induced pseudo-Cushing's syndrome. Lancet. 1993;341:676-7.

(32) Lindenbaum J. Hematologic complications of alcohol abuse. Sem Liver Dis 1987; 7: 169-181.

(33) Liu YK. Effects of alcohol on granulocytes and lymphocytes. Sem Hematol 1980; 17: 130-136.

(34) Levine RF, Spivak LJ, Meagher RC, Sieber F. Effect of ethanol on trombopoiesis, $\mathrm{Br} \mathrm{J}$ Henatol 1986;62:345-354.

(35) Cowan DH. Effects of alcoholism on hemostasis. Sem Hematol 1980; 17: 137-147.

(36) Peris P, Pares A, Guanabens N, Pons F, Martinez de Osaba MJ, Caballeria J, et al. Reduced spinal and femoral bone mass and deranged bone mineral metabolism in chronic alcoholic patients. Alcohol and Alcoholism 1992; 26:619-625.

(37) Peris P, Parés A, Guañabens N, del Río L, Pons F, Martínez de Osaba MJ, et al. Bone mass improves in alcoholics after two years of abstinence. J Bone Miner Res 1994; 9:1607-1612.

(38) Garro AJ, Lieber CS. Alcohol and cancer Annu Rev Pharmacol Toxicol 1990; 30: 219-249.

(39) Longnecker M. Alcohol beverage consumption in relation to risk of breast cancer: Meta-analysis and review. Cancer Causes Control 1994; 5: 73-82.

(40) Jones KL. Fetal alcohol syndrome. Pediatr Rev 1986; 8: 122-126. 